\title{
Rapid Diagnosis of Tubercular Bacilli in Sputum Cytology
}

\author{
Rajat Kumar $^{1 *}$, Khushbu Kumari ${ }^{1}$, K D Kulkarni ${ }^{2}$, S.S Hiremath ${ }^{1}$, Prabhu M H ${ }^{1}$ \\ ${ }^{1}$ Deptt. Of Pathology, SNMC \& HSK Hospital, Bagalkot, India \\ ${ }^{2}$ Deptt. Of Pulmonary Medicine, SNMC \& HSK Hospital, Bagalkot, India
}

\begin{abstract}
Background: Tuberculosis is a most common infectious disease which is still remains a leading cause of adult mortality. There are various methods for the diagnosis of tuberculosis, such as sputum examination of tubercular bacilli by Ziehl-Neelsen(ZN) staining, demonstration of tubercular bacilli by Auramine-O, Papanicolaou(Pap) stain staining and culture in LJ medium.
\end{abstract}

Aim: The aim of present study was to evaluate the diagnostic utility of Pap stain, Auramine-O and ZN stain in rapid diagnosis of pulmonary tuberculosis in sputum cytology.

Material \& Methods: Study was done on Sputum of clinically suspected case of pulmonary tuberculosis from June 2017 to August 2017. On all received sample Z N, Pap, Auramine-O staining done.

Result: Among clinically suspected 150 patients. Tuberculosis was diagnosed in 74 patients. Pap shows 49 (66.21\%), Auramine-O shows 42(56.75\%); ZN shows 35 (47.29\%) cases positive.

Conclusion: Evening rise of Temperature, Weight Loss, and Cough more than 2 weeks, CXR reports are the main evidence to suspect a case of Tuberculosis. Males are more affected than female and more in case of seropositive patient. Pap is slightly more sensitive than Auramine-O in diagnosis of Pulmonary tuberculosis in Fluorescent Microscopy.

Keywords: Pap, Auramine-O, Sputum, ZN stain, Fluorescent Microscopy.

\section{Introduction}

Mycobacterium Tuberculosis (MTB) is most common communicable disease in low socioeconomic population. It is most common in developing countries like India.

Causative organism was discovered more than 100 years ago and highly effective drugs and vaccine are available, still it is prevalent in our society. ${ }^{[1]}$

Koch first described the tubercle bacilli in 1882. [ ${ }^{1]}$ Mycobacteria are now known to comprise a large group of acid-fast, alcohol-fast, aerobic or micro-aerophilic, nonspore forming, non-motile bacilli. ${ }^{[1]}$

World Health Organization (WHO) statistics as of 2011 estimates gives global incidence of 8.7 million cases, about 2.2 million from India. About $40 \%$ of Indian population is infected with tuberculosis. ${ }^{[2]}$

In developing countries like India with deficit of resources compared to high TB burden, culture facility is not adequately available. Hence most of the TB cases are diagnosed based on Sputum Smear Microscopy (SSM).

${ }^{[5]}$ Hence, Rapid and effective methods are required for detection of Tubercle bacilli in sputum in limited resource setup.
Many laboratories prefer Rhodamine Auramine method of staining AFB over carbol fuchsin stain because the fluorescing bacilli are more readily detected. Auramine stained smears are scanned under low magnification than fuchsin stained smears, thus permitting a large area of the smear to be examined in shorter period of time. Diagnosis of pulmonary tuberculosis by demonstration of Acid Fast Bacilli (AFB) in sputum smears by $\mathrm{ZN}$ staining is known to be economical and less time consuming. ${ }^{[6]}$

Papanicolaou stain is widely used in routine cytological evaluation of samples derived from the respiratory tract. Its value in the fluorescence microscopic diagnosis of various pulmonary infectious diseases is well established. ${ }^{[7]}$ In Pap stained smears the slightly curved, beaded tubercle bacillus may be readily identified. ${ }^{[8]}$ Limited data are available regarding efficacy of different types of stain especially Pap stain in detection of tubercular bacilli.

So, we studied the efficacy of different types of stain i.e $\mathrm{ZN}$, PAP, Auramine-O in the diagnosis of tuberculosis in sputum sample.

\section{Material \& Methods}

Study was done on Sputum of clinically suspected case of pulmonary tuberculosis from June 2017 to August 2017 in 
Department of Pathology, S N Medical College \& HSK Hospital, Bagalkot. Study was done on clinically suspected cases. Usual clinical presentation is fever, cough, sputum and weight loss.

Sputum collection: Sputum of 24 h duration was collected in dry, sterile bottles.

Three smears were prepared from each sample (Two dry and one wet smear). Three smears were stained by routine Ziehl-Neelsen (ZN) method and other by fluorescence stain Auramine-Rhodamine and Pap (Papanicolaou) stain.

Ziehl-Neelsen stain shows-AFB stain pink curve/straight beaded rods against blue background.

Auramine-O stain shows-AFB appears as bright reddishyellow fluorescing rods against a dark background and

Papanicolaou stain fluorescent bacilli shows- slender, often beaded, yellow-green, straight or slightly curved rods of relatively uniform length against a dark background.

Grading of PAP \& Auramine-O smears done as: $>10$ bacilli/field $=2+; 2-9$ bacilli/field $=1+; 10-99$ bacilli $/ 100$ fields $=+; 1-9$ bacilli/ 100 fields $=$ Scanty; No bacilli in 100 fields $=$ Negative. ${ }^{[14]}$
Positive control smears were prepared from cultured colonies of Mycobacterium tuberculosis (MTB) and negative control salivary smears were prepared from saliva sediment of a healthy individual for both the staining methods.

$\mathrm{ZN}$ smears were graded using 100x oil immersion objective as per RNTCP guidelines. ${ }^{[13]}$ Scanty $=1-9$ AFB in100 oil immersion fields; Grade $1+=10-99$ AFB in 100 oil immersion fields; Grade $2+=1-10$ per oil immersion field an atleast 50 fields; Grade $3+=10$ or more AFB per field in at least 20fields; Negative $=$ no AFB in 100 fields.

\section{Result}

Among clinically suspected 150 patients. Tuberculosis was diagnosed in 74 patients. Males are more infected than female. PAP shows 49 (66.21\%), Auramine-O shows 42(56.75\%); ZN shows $35(47.29 \%)$ cases positive. Of the 150 cases evaluated all were above 16 years of age with a range of 16-80 years. Among clinically suspected 150 patients. Tuberculosis was diagnosed in 74 patients. The peak incidence was seen in the second-third decades.

RESULT (Total case $=150$, Positive case $(n)=74$ )

\begin{tabular}{|c|c|c|}
\hline Stain & Positive cases (n) & $37.83 \%$ \\
\hline Pap & 28 & $32.43 \%$ \\
\hline Auramine-O & 24 & $29.72 \%$ \\
\hline ZN & 22 & \\
\hline
\end{tabular}

*FMP=Fluorescent Microscopy

Result compared from different study

\begin{tabular}{|c|c|c|c|}
\hline Stain & Mani et al & Madhusudhan et al & Present Study \\
\hline ZN & $16.16 \%$ & $27.65 \%$ & $29.72 \%$ \\
\hline Pap & $33.35 \%$ & $24.90 \%$ & $37.83 \%$ \\
\hline Auramine-O & $31.34 \%$ & $27.44 \%$ & $32.43 \%$ \\
\hline
\end{tabular}

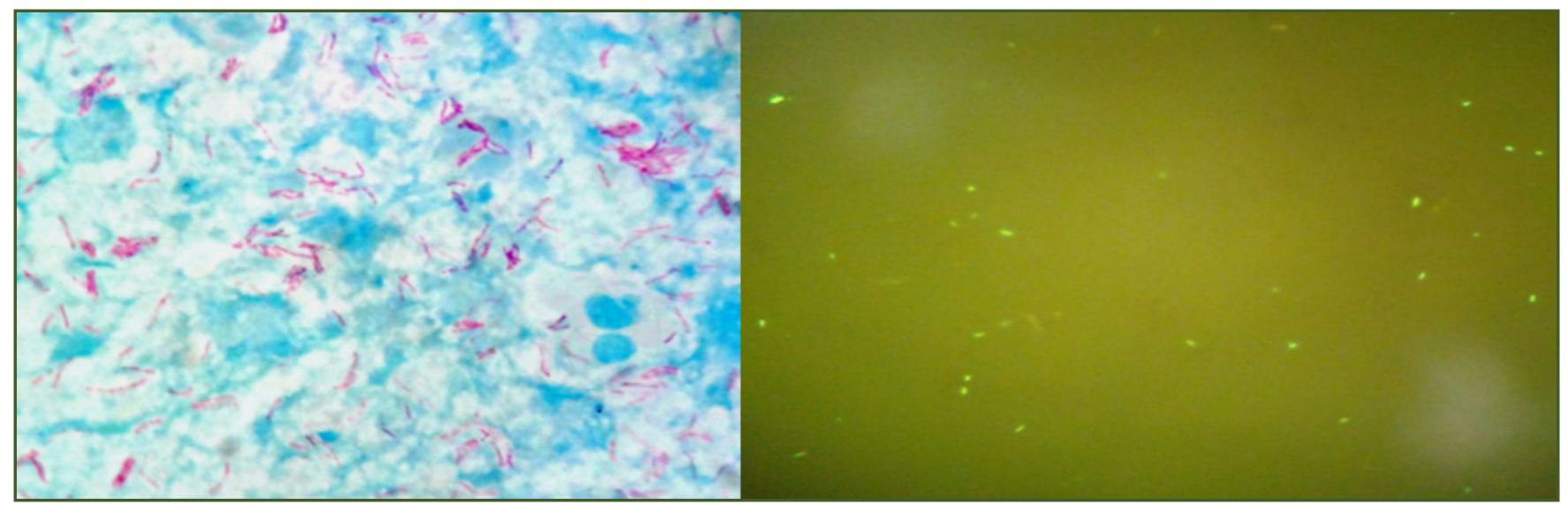

Fig. I: AFB Positive (ZN stain 100X), Fig.IIAuramine-0 (FMP*) 40X. 


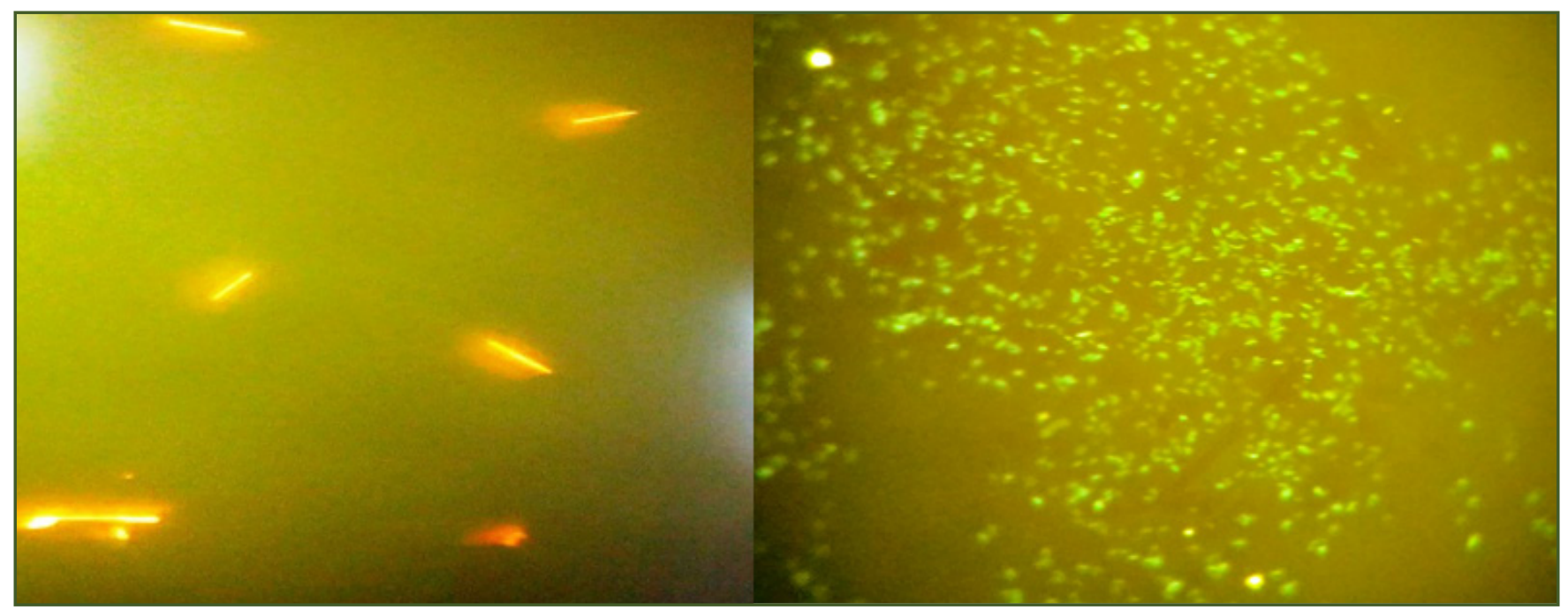

Fig. IIIPap Stain (FMP*) 40X, Fig. IV Auramine-0 (FMP*) 20X.

\section{Discussion}

Recent global statistics reveal 8.6 million incident cases of Tuberculosis (TB) with 1.3 million deaths. ${ }^{[3]}$ This TB crisis has further escalated with triggering of the HIV epidemic and emergence of multidrug-resistant TB. ${ }^{[4]}$

Tuberculosis commonly affects lungs but can also be extra-pulmonary. Hence microscopic examination of sputum for detection of Acid Fast Bacilli (AFB) is of utmost importance. Early detection can prevent further complication. ${ }^{[4]}$

An advantage of fluorescence microscopy lies in the ease with which the bacilli can be detected due to the color contrast, allowing for a larger area of the smear to be scanned at lower magnification when compared to conventional Z-N staining. Since the Pap stain is routinely used in cytology, it saves the time and material that would be involved in any extra staining and also avoids the use of toxic or carcinogenic substances such as phenol and rhodamine, which are used in A-R staining method. ${ }^{[7]}$

In India prevalence of tuberculosis is 400 per 100,000 population. ${ }^{[12]}$

Diagnostic utilization of UV-induced fluorescence for detection of infective pathogens was first described by Graham (1983) ${ }^{[16]}$ and later Mann (1983). ${ }^{[17]}$

Ghali et al. (1984). first demonstrated auto fluorescence of pneumocystic carinii in Pap stained smears. Their results indicated eosin to be responsible for the auto fluorescence. [9] Its value in the fluorescence microscopic diagnosis of various pulmonary infectious diseases is well established. ${ }^{[3]}$

Küpper et al. reported significant difference between the sensitivities of Pap fluorescence (FMP), A-R fluorescence
(FMA) and Z-N staining respectively for their ability to detect atypical Mycobacterium kansasi in cytological samples. ${ }^{[15]}$

Our results were similar with Kupper et al. and Lunawat PP et al. in their studies, which also found AF stain was more sensitive in comparison with $\mathrm{AR}$ and $\mathrm{ZN}$ stain for the diagnosis of tubercular bacilli. ${ }^{[11]}$

An advantage of fluorescence microscopy lies in the ease with which the bacilli can be detected due to the colour contrast, allowing for a larger area of the smear to be scanned at lower magnification when compared to conventional Z-N staining. ${ }^{[11]}$

The Pap stain is routinely used in cytology. It saves time and material that would be involved in any extra staining and also avoids the use of toxic or carcinogenic substances such as phenol and Rhodamine, which are used in AR staining method. It also offers the advantage of simultaneous identification of other agents such as pneumocystic carinii, aspergilla and candida in the material obtained primarily for the diagnosis of $\mathrm{TB}$, especially in immunocompromised patients.

\section{Conclusion}

Fluorescence microscopy is a useful, rapid, and reliable and is quite economical tool for the diagnosis of mycobacterium bacteria in specimens for AFB in comparison to $\mathrm{ZN}$ staining because of the higher sensitivity and rapidity of the fluorochromes technique compared to $\mathrm{ZN}$.

The PAP stain is routinely used in cytology, it saves the time and material that would be involved in any extra staining and also avoids the use of toxic or carcinogenic substances, such as phenol and Rhodamine, which are used in AR staining method. 
However, this method should be used primarily as a screening technique, and every diagnosis should be confirmed by microblogical culture.

The method used in the present study enables in rapid identification of these infective sources and hence prevent further transmission and is thus important in TB control.

\section{References}

1. Krishna M, Kumar A; Autofluorescence in Pap stain in the sputum of suspected pulmonary tuberculosis and compare with other AFB stains. J. Evolution Med. Dent. Sci. 2016;5(16):755-757, D

2. Global tuberculosis control 2012, WHO, Geneva, 2012.

3. Global Tuberculosis Report;WHO;Geneva; 2013.

4. Connolly C, Davies GR, Wilkinson. D. Impact of the human immunodeficiency virus epidemic on mortality among adults with tuberculosis in rural South Africa, 1991- 1995. Int J Tuberc Lung Dis 1989;2(11):919-25.

5. Madhusudhan NS, Amirthalingeswaran G; Comparison of ZN stain (RNTCP) Versus fluorescent microscopy and modification of cold stain to detect acid fast bacilli from sputum sample; International Journal of Contemporary Medical Research 2016;3(4):968-971.

6. Huebner RE, Good RC, Tokars JI; Current practices in mycobacteriology: results of a survey of state public health laboratories; J Clin Microbiology 1993;31:771-5.

7. Küpper T, Gore C, Gore R, Wehle K, Marzahn S, Pfi tzer P. Fluorescence microscopy of mycobacteria in pleural effusions; Cytopathology 1999;10:303-7.

8. Kupper TH, Wehle K, Pfitzer P; Fluorescence microscopy of Papanicolaou stained preparations; Acta Cytol 1994;38(4):64.
9. Ghali VS, Garcia RL, Skolom J; Fluorescence of Pneumocystis carinii in Papanicolaou smears. Hum Patho 1984;15(10):907-9.

10. Küpper T, Steffen U, Wehle K, Richartz G, Pfitzer $\mathrm{P}$; Morphological study of bacteria of the respiratory system using fluorescence microscopy of Papanicolaou stained smears with special regard to the identification of Mycobacteria sp. Cytopathology 1995;6(6):388-402.

11. Lunawat PP, Mhapuskar AA, Ganvir SM, Hazarey VK, Mhapuskar MA, Kulkarni D; Detection of acid fast bacilli in saliva using papanicolaou stain induced fluorescence method versus fl uorochrome staining: An evaluative study; J Int Oral Health 2015;7(7):115-120.

12. Mandal AK, Chaudhary S; Tuberculosis In; Mandal AK, Chaudhary S, editor. Text book of pathology, Ist ed. New Delhi; Avichal Publisher; 2010:70-75.

13. RNTCP Central TB Division Manual for Laboratory Technicians. New Delhi, India Directorate General of Health Services, Ministry of Health and Family Welfare; 2nd Ed, $2005 ; 22,50$.

14. Weyer K, Kantor I, Kim SJ; Microscopy.In: Laboratory Services in Tuberculosis Control;Ist edition; New Delhi; A.I.T.B.S; 2002. p. 79-111.

15. Küpper T, Wehle K, Marzahn S, Pfitzer P; The cytologic diagnosis of Mycobacterium kansasi tuberculosis by fluorescence microscopy of Papanicolaou-stained specimens; Cytopathology 1995;6(5):331-8.

16. Graham AR; Fungal autofluorescence with ultraviolet illumination. Am J Clin Pathol 1983;79:231-4.

17. Mann JL; Autofluorescence of fungi: An aid to detection in tissue sections. Am J Clin Pathol 1983;79(5):587-90.

*Corresponding author:

Dr. Rajat Kumar, Deptt. Of Pathology, SNMC \& HSK Hospital, Bagalkot. Pin-587102. INDIA

Phone: +91 8004943886

Email: rajat.kumar5060@gmail.com

Financial or other Competing Interests: None. 